Wright State University

CORE Scholar

International Symposium on Aviation

International Symposium on Aviation

Psychology - 2007

Psychology

2007

\title{
An Ecological Approach to Support Pilot Terrain Awareness After Total Engine Failure
}

Floris Sjer

Clark Borst

Max Mulder

M.M. van Paassen

Follow this and additional works at: https://corescholar.libraries.wright.edu/isap_2007

Part of the Other Psychiatry and Psychology Commons

\section{Repository Citation}

Sjer, F., Borst, C., Mulder, M., \& van Paassen, M. (2007). An Ecological Approach to Support Pilot Terrain Awareness After Total Engine Failure. 2007 International Symposium on Aviation Psychology, 657-663. https://corescholar.libraries.wright.edu/isap_2007/23

This Article is brought to you for free and open access by the International Symposium on Aviation Psychology at CORE Scholar. It has been accepted for inclusion in International Symposium on Aviation Psychology - 2007 by an authorized administrator of CORE Scholar. For more information, please contact library-corescholar@wright.edu. 


\title{
AN ECOLOGICAL APPROACH TO SUPPORT PILOT TERRAIN AWARENESS AFTER TOTAL ENGINE FAILURE
}

\author{
Floris Sjer, Clark Borst, Max Mulder, M. M. van Paassen \\ Delft University of Technology \\ Faculty of Aerospace Engineering \\ Control and Simulation Division \\ Delft, The Netherlands
}

Terrain awareness enhancing avionics, such as Synthetic Vision Systems and the Enhanced Ground Proximity Warning System, have been developed to reduce the number of controlled flight into terrain accidents. The protection these systems offer, however, is far from optimal. Synthetic Vision Systems only provide pilots with perceptual data, and leave all cognition and interpretation of data to the pilot. With Enhanced Ground Proximity Warning Systems the opposite is true. Here, cognition is hidden in the system and pilots are confronted with compelling advisories and commands. This paper presents a display system, the Emergency Landing Guidance System, which visualizes the functional meaning of surrounding terrain, adopting an ecological interface design approach. The potential benefits of this approach are demonstrated with the case of locating and approaching a suitable landing spot in the situation of a sudden complete engine failure. To evaluate the amount of pilot terrain awareness supported by the new display, an experiment was conducted in a fixed-base flight simulator. Results show that the new display supports pilot terrain awareness much better than present terrain avoidance systems, especially regarding awareness involving the higher levels of cognitive processing. Pilots better understand the meaning of the terrain topology in relation to their goals and constraints.

\section{Introduction}

Pilot terrain awareness supported by existing systems, such as the Enhanced Ground Proximity Warning System (EGPWS) and Synthetic Vision System (SVS) can be improved by mapping the internal aircraft constraints, imposed by the aircraft's performance, onto the external terrain constraint (Borst, Suijkerbuijk, Mulder and Van Paassen, 2006). For this paper, a new display was developed and evaluated using the Ecological Interface Design (EID) paradigm (Vicente and Rasmussen, 1992), based on the terrain awareness abstraction hierarchy (AH) (Borst et al, 2006), to support pilots in coping with the situation of a total engine failure. The new display, the Emergency Landing Guidance System (ELGS), visualizes the functional meaning of surrounding terrain in terms of locating and approaching suitable landing spots after sudden complete engine failure.

General Aviation (GA) was chosen as the application domain, since this community has picked up technologies like the SVS and EGPWS to increase pilot situational awareness (SA) and, more importantly, safety. The case study of a total engine failure was chosen based on several accident reports that showed that awareness about the terrain and aircraft capabilities is critical in this situation (Khatwa and Roelen, 1999).

The structure of this paper is as follows. First, the constraints on each level of the AH in the situation of a total engine failure are described. Second, the design of the ELGS interface is elaborated. Third, an experimental evaluation of the ELGS is described. Finally, the experiment results are discussed.

\section{Constraints After Total Engine Failure}

The terrain awareness AH is shown in Figure 1. In the situation of a total engine failure the engine thrust force, a constraint on the generalized function level, is zero. Hence, with this altered constraint, some of the goals defined in the AH cannot be achieved. Whereas the AH itself does not change in case of a total engine failure, the constraints and priority measures of the work domain will be different than in a situation where all aircraft systems work fine.

\section{Functional purpose}

When considering the work domain in a situation of a total engine failure, the focus will be on the safety, because an emergency landing needs to be conducted for which productivity and efficiency are relatively less important. The purpose is to land as-safe-aspossible on suitable and safe terrain.

\section{Abstract functions}

In case of a total engine failure, the thrust is zero and pilots are likely to try to maximize the aircraft's range, thereby maximizing the number of places where an emergency landing can be conducted. For this purpose the aircraft drag and speed need to be 
controlled such that the absolute value of the total energy rate is minimized.

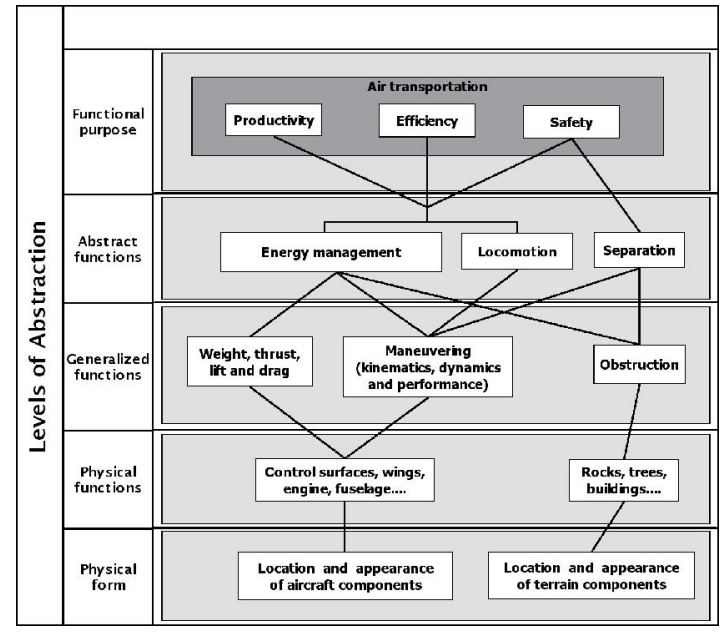

Figure 1. Abstraction Hierarchy with 'means-ends' links for terrain awareness (Borst et al, 2006)

Assuming that an optimum gliding flight with maximum range is conducted at a constant speed, minimizing the total energy rate reduces to minimizing the potential energy rate or, equivalently, the descent rate. This, however, only holds up to the moment where the pilot has chosen the suitable emergency landing spot. After the choice has been made, the required total energy rate to arrive exactly at the chosen emergency landing spot is likely to be different from the minimum absolute value. For example, if the distance to the landing spot is much smaller than the maximum glide range, the energy rate must be higher than the minimum absolute value. Therefore, pilots will have to determine what this required total energy rate is in order to prevent overshooting or not reaching the desired landing spot at all.

While doing so, both the potential and kinetic energy impose an important constraint. The minimal potential energy corresponds to safe terrain separation and the minimal kinetic energy corresponds to the aircraft's safe touchdown speed. Therefore, in case of a total engine failure the energy management problem is to reduce the current total energy to the total energy required for the landing spot by choosing a proper potential energy rate.

\section{Generalized functions}

To maximize the number of places where an emergency landing can be conducted, the aircraft needs to be controlled in such a way that the range is maximized. This maximum range can be described by the optimum glide of an aircraft (Ruijgrok, 1996).
The glide range is maximal when performing a gliding flight without turns. To reach a landing area, however, it may be required to make one or more turns. Furthermore, the maximum glide range also depends on the aircraft configuration, such as the flap setting and the landing gear position. These devices decrease the glide ratio (or aerodynamics efficiency) by increasing the aircraft drag. As a result, the glide range will be reduced.

At the time a total engine failure occurs, the airspeed is likely to be different from the airspeed at which an optimum glide is conducted. If the airspeed is higher than the optimum airspeed of the glide, a pull-up maneuver is conducted to exchange the excess in kinetic energy into potential energy. If the airspeed is lower than the optimum airspeed of the glide, a pushover maneuver is initiated to exchange altitude into kinetic energy.

\section{Physical functions}

At this level of abstraction, the flap setting and landing gear position are important to control the aircraft drag and hence the glide performance. The terrain's physical function remains the same as in Figure 1, however, now the lack of rocks, trees and buildings defines a potential landing area.

\section{Physical form}

The constraints on this level remain the same as shown in the abstraction hierarchy (Figure 1).

\section{Supporting Skill, Rule and Knowledge Based Behavior}

Nowadays, after a total engine failure a GA pilot will use the out-of-the-window view to identify suitable landing areas. This can be categorized as Skill Based Behavior (SBB) and is known from practice to be unreliable and to be affected by atmospheric visibility conditions. In terms of Rule Based Behavior (RBB), a pilot will use the altimeter and navigational maps to determine the altitude above suitable landing areas on the surrounding terrain. Based on that information, a pilot will apply a strategy to reach a selected landing area. The process of scanning maps and mentally calculating landing spots within reach may lead to high levels of workload. Within the time frame of looking for suitable landing areas and figuring out how to get there, there is virtually no time left for Knowledge Based Behavior (KBB).

To support SBB in case of a total engine failure, the interface should help the pilot to identify suitable 
landing spots given the aircraft's glide range. On the level of RBB, the interface should make it possible to recognize situations and formulate shortcuts by an ifthen mapping between familiar perceptual cues and appropriate actions. For example, a perceived terrain obstruction on the glide trajectory triggers the pilot to make turns to fly around the obstacle. Furthermore, pilots should also be supported in terms of managing the aircraft configuration. To support KBB, the interface should make it possible to reason at abstract levels by explicitly considering the goals. For example, consider energy management and obstruction versus locomotion. By keeping the safety goal in mind, a pilot should be able to build strategies on how to glide along trajectories that have the least amount of obstructions.

\section{Interface Design}

It was chosen to map the goals and structure of the constraints in the $\mathrm{AH}$ as an interface overlay of an EGPWS Terrain Awareness Display (TAD).

\section{Informational content}

Aircraft performance. To show the internal aircraft performance constraints in the situation of a total engine failure, a three-dimensional glide path mesh (Figure 2) was projected onto the terrain. The curved parts of glide path mesh are turns with a maximum bank angle of 45 degrees. The intersection of the glide mesh and the terrain indicates the aircraft's maximum range for the particular configuration. Landing spots within the mesh are those that can be reached. The 45 degrees turns serve as a safety margin in case a turn is needed to fly towards a certain landing spot. Turning with a bank angle less than 45 degrees of course increases the aircraft's range, but turning with higher bank angles have the opposite effect.

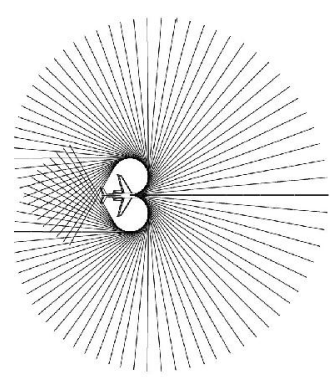

Top view

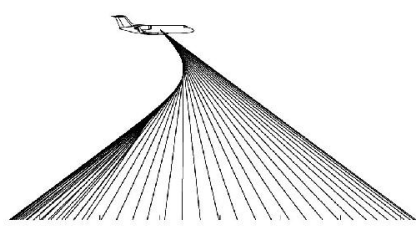

Side view
Figure 2. Top view and side view of the glide path mesh for a particular configuration of the Cessna Citation 500

Terrain separation. The present and future height above the terrain is indicated by presenting the vertical spacing between the glide mesh and the terrain in terms of color coding as shown in Table 1. The red color indicates where the terrain rises above the glide mesh.

Table 1. Ground proximity colors of the ELGS

\begin{tabular}{ll}
\hline \hline Color & Ground proximity interval $(\mathrm{ft})$ \\
\hline red & $\geq 0$ \\
yellow & {$[-500,0)$} \\
brown & {$[-1000,-500)$} \\
green & {$[-1500,-1000)$} \\
dark green & {$[-2500,-1500)$} \\
black & $<-2500$ \\
\hline
\end{tabular}

Landing spot detection. Currently, the landing spots in the terrain elevation database are only detected by the elevation rate or 'terrain roughness'. An area suitable for landing, that is, a smooth area in the terrain database, is indicated on the TAD. The area can be reached as long as it lies within the aircraft's glide range.

Pilots do not yet know, however, how much altitude they need to lose over the range to arrive at the landing area without overshooting it. Therefore, the landing spots are given a specific color that corresponds to a certain aircraft configuration to safely arrive at the landing spot, see Table 2 and Figure 3. The aircraft configurations apply to the Cessna Citation 500.

Table 2. Landing spot colors indicating the configuration of the Cessna Citation 500

\begin{tabular}{llll}
\hline \hline Color & Flaps [deg] & Gear & $V_{\text {TAS }}[\mathrm{kts}]$ \\
\hline bright red & 0 & $\mathrm{Up}$ & 130 \\
bright orange & 15 & $\mathrm{Up}$ & 115 \\
bright green & 15 & Down & 115 \\
bright light blue & 40 & Down & 100 \\
bright blue & Turn needed & & \\
\hline
\end{tabular}

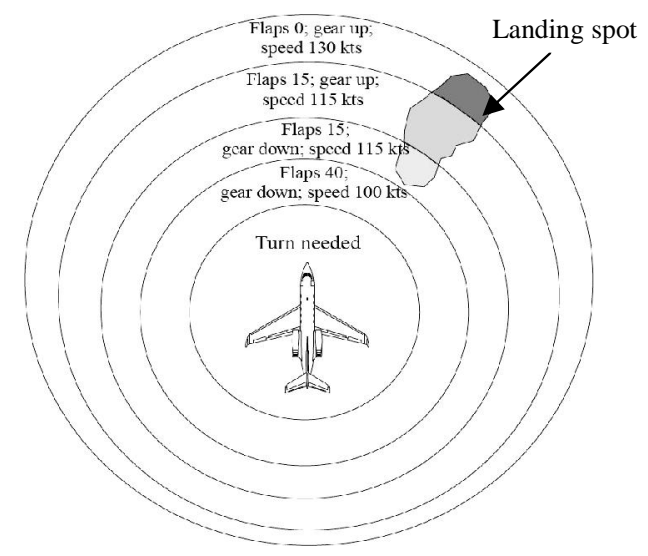

Figure 3. Plan view of the glide path mesh footprints, corresponding to the Cessna Citation 500 configurations, mapped onto a landing spot 


\section{Visual form}

The ELGS interface overlay consists of two layers projected on top of the terrain: 1) the glide path mesh with ground proximity colors and 2) the color-coded landing spots. Salience is used to distinguish the two layers, where the landing spots are colored more brightly than the ground proximity colors to make them stand out more. A screenshot and a conceptual representation of the ELGS overlay are shown in Figures 4 and 5, respectively. How to use the ELGS interface overlay can best be explained by an example situation shown in Figure 5.

In this figure, the ground proximity areas (a result of mapping the glide path mesh onto the terrain) are indicated by I, II, III and IV, where I shows where the terrain rises above the glide mesh and is colored in red (see Table 1). This basically defines the safety margin within which the aircraft can fly without colliding with terrain. As the aircraft descends, the safety margin gets smaller. From Figure 5 it can also be seen that inside the safety margin there are two reachable landing spots, that is, A and B. Landing spot $C$ lies outside the safety margin and therefore cannot be reached. With two reachable landing spots $\mathrm{A}$ and $\mathrm{B}$, the pilot has to make a decision which one is most preferable to land. Landing spot B lies closest to the aircraft, but the energy excess is so large that the pilot has to make additional turns to lose the excess in potential and kinetic energy. If the pilot chooses to land on $\mathrm{B}$, making a turn to the left will result in a terrain collision. Landing spot $A$, however, can be reached by a straight gliding flight with a flaps 15 and gear down configuration. Based on that information, a pilot could choose to make a straight gliding flight towards landing spot A.

\section{Experiment}

\section{Method}

Subjects. Nine licensed GA pilots, all males with an average age of 25 and an average of 489 flying hours, participated in the experiment. To eliminate the pilots' skills as a variable as much as possible from the experiment results, a homogeneous group of pilots was selected, that had enjoyed the same pilot training and limited experience in terms of flight hours.

Task. The terrain topologies used in the experiment were setup in such a way that always three emergency landing spots were shown. Pilots were instructed to perform an emergency landing on one of the three spots. They were also instructed to touch down with as much roll out margin in front of them as possible.
Apparatus. The experiment was conducted in a fixedbase flight simulator, consisting of a cabin mockup, situated in a darkened noise-free room. The cabin has two 18" TFT LCD monitors. One is situated in front of the pilot, which was used to display the SVS.

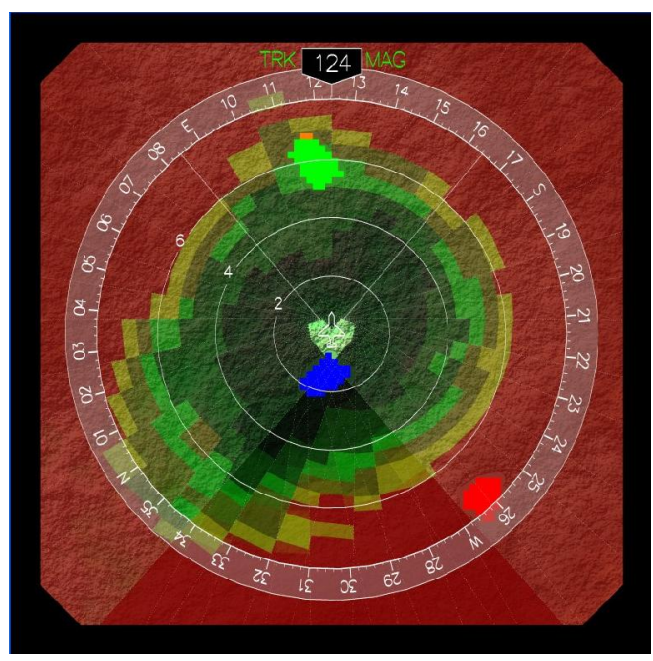

Figure 4. ELGS screenshot

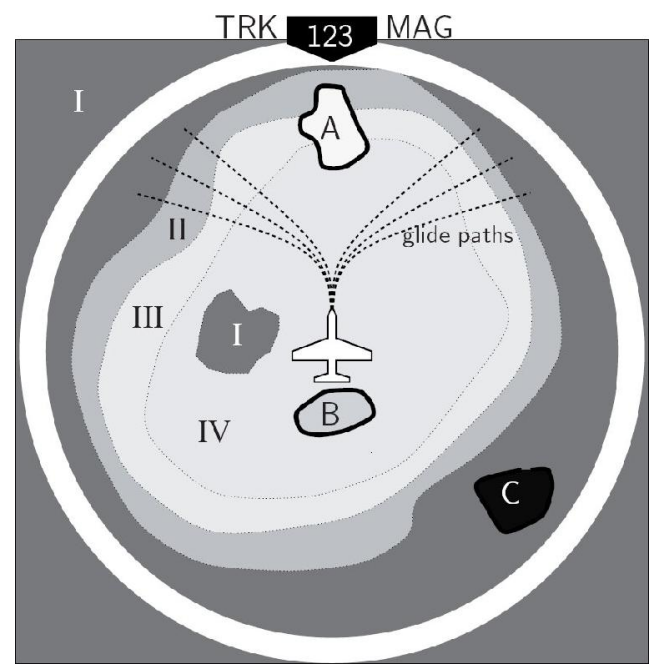

Figure 5. ELGS conceptual representation

The other display is situated to the left of the pilot and showed the Horizontal Situation Display (HSD) and the engine instruments.

Aircraft characteristics and experiment conditions. The aircraft model used in the simulation is a 6-degree of freedom non-linear Cessna Citation 500 model. The aircraft model was trimmed at an altitude of 2500 meters with a velocity of 150 knots in an ISA standard atmosphere with no wind or turbulence present. 
Independent variables. There were two independent variables in the experiment, the display configuration and the experiment terrain. The display configuration had 3 levels and the experiment terrain had 9 levels, creating a total of 27 experiment conditions.

Three display modes were configured, MAP, EGPWS and ELGS. MAP featured a Primary Flight Display (PFD) and a top-view of the terrain on which a color map illustrated the elevations of the terrain on the HSD. The landing spots were indicated on the map. EGPWS featured a SVS and a HSD overlaid with EGPWS colors. Besides EGPWS color-coding, this display configuration also included an EGPWS forward looking flight-path prediction along with aural and visual caution/warning messages. ELGS featured a SVS and an HSD with the ELGS logic and color coding.

Nine mountainous virtual terrains were created. Each terrain featured three landing areas. The location and height of the landing areas with respect to the aircraft's state at the moment of the engine failure was chosen such, that: 1) one of the areas is unreachable or only reachable in an optimal gliding flight, 2) one is reachable by performing one turn followed by a straight gliding flight, and, 3) one is reachable but a large amount of excess energy needs to be lost through extra turns.

The difficulty of each scenario was kept at an equal level as much as possible and the scenarios were flown in each display configuration an equal number of times.

Dependent measures. The dependent measures were: 1) performance, in terms of grading the chosen landing spot and touchdown point, and, 2) terrain awareness, in terms of perception, comprehension, projection, and metacognition (McGuiness, 1999). The landing spot choice was graded ' 1 ' for choosing the most suitable landing spot and a ' 3 ' for least suitable. The touchdown point was graded ' 1 ' for landing at the start of the spot, a ' 2 ' for in the middle and a ' 3 ' for at the end.

Terrain awareness was measured using interruptive query probing and a post-run questionnaire (Endsley, 2003). The interruptive query probing was done in the form of short questionnaires of six questions that were aimed at different levels of awareness. Questions at the first level, that is, perception, were like: "What is your indicated airspeed?" or "Where are suitable landing spots with respect to your current heading?”. At the second level, comprehension, questions like: "Which landing spot cannot be reached?" were asked. The third level, projection, was tested by questions like: "What strategy is appropriate to reach your selected landing spot?”. To quantify these first three levels, a model was created to specify whether pilots were incorrect, far off, almost correct or correct. These levels were graded with $0,3,6$ or 9 , respectively. The thresholds between the levels to which the given answer was to be directed, were partly determined by expert subjects and partly by comparing the correct answer to a question.

The fourth level of terrain awareness metacognition, was measured through a confidence interval. Next to each question the pilots indicated on this interval their self-confidence, with a number ranging from 0 to 10,0 being very unsure and 10 being very sure. Of the indicated values a z-score was taken, with which the pilots were graded $0,1,2,3,6$ or 9 for the metacognition. The determination of the metacognition grade was done through a combination of the self-confidence interval and the grade obtained for the question itself. With these two numbers Table 3 was used.

Table 3. Grade of metacognition

\begin{tabular}{|c|c|c|c|}
\hline & $\begin{array}{c}\text { absolutely sure } \\
z>0.33\end{array}$ & $\begin{array}{c}\text { fairly sure } \\
0.33>z>-0.33\end{array}$ & $\begin{array}{c}\text { unsure } \\
z<-0.33\end{array}$ \\
\hline correct: grade 9,6 & 9 & 6 & 3 \\
\hline incorrect: grade 3,0 & 0 & 1 & 2 \\
\hline
\end{tabular}

Procedure. The experiment consisted of two phases: training and measurement. The experiment phase consisted of nine experiment runs, where each display mode was used three times. Figure 6 shows the time-line of an experiment run.

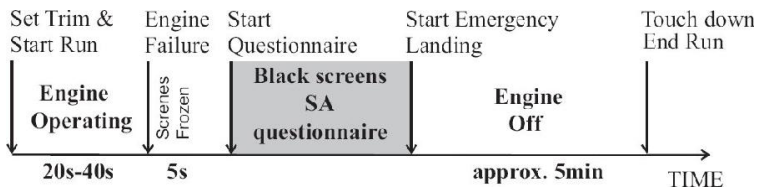

Figure 6. Time-line of an experiment run

\section{Results and Discussion}

The analysis of the experiment measurements was done using Analysis of Variance (ANOVA). Post-hoc analyses were performed using Student-NewmanKeuls (SNK, $\alpha=0.05$ ) range tests. For all results, ANOVA analysis showed no significant effects of the terrain on the dependent measures. 


\section{Performance}

The performance in terms of landing spot choice and touchdown point both are highest (lowest grade) for ELGS (Figure 7). The figure shows that when pilots were flying with ELGS, they made better landing spot choices $\left(\mathrm{F}_{2,46}=4.657, \mathrm{p} \leq 0.05\right)$ and their touchdown point was also better $\left(\mathrm{F}_{2,46}=5.344\right.$, $\mathrm{p} \leq 0.01$ ). Post-hoc analysis showed that the pilots performed better in MAP than in EGPWS, although the difference was not significant.

\section{Terrain awareness}

The results of the terrain awareness measurements are shown in Figures 8 and 9. Analysis showed that the display mode had a highly significant effect on the projection $\left(\mathrm{F}_{2,111}=6.235, \quad \mathrm{p} \leq 0.01\right)$ and metacognition $\left(\mathrm{F}_{2,111}=8.466, \mathrm{p} \leq 0.01\right)$ levels, and a borderline significance on the comprehension level $\left(\mathrm{F}_{2,111}=3.067, \mathrm{p} \leq 0.1\right)$. The increase in terrain awareness is very clear for the projection and metacognition level.
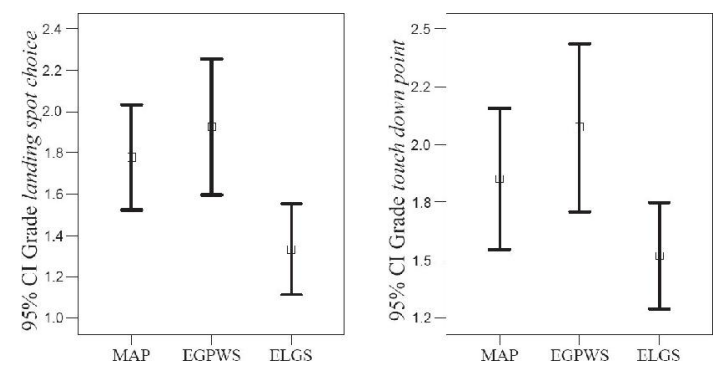

Figure 7. Means and 95\% confidence intervals of the display mode on the grade for performance
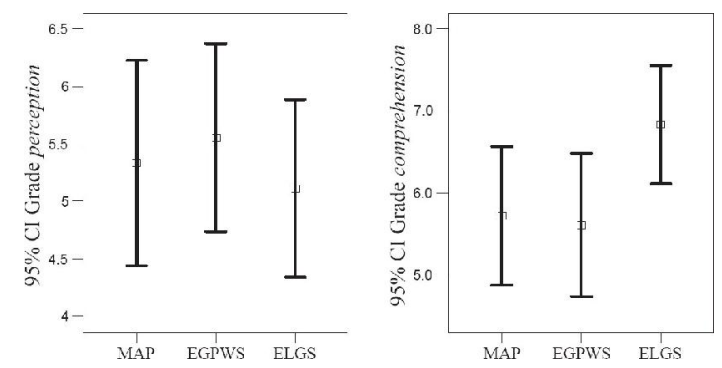

Figure 8. Means and 95\% confidence intervals of the display mode on the grade for perception and comprehension
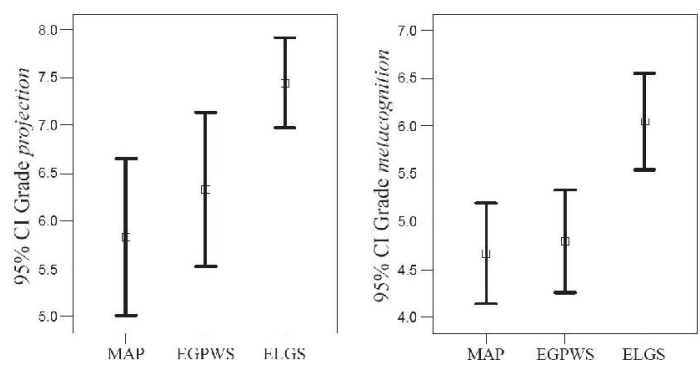

Figure 9. Means and 95\% confidence intervals of the display mode on the grade for projection and metacognition

\section{Discussion}

The goal of the design of the ELGS, using Ecological Interface Design, was to investigate the terrain awareness achieved by it. The experiment results show that pilot terrain awareness in the situation of a complete engine failure is better supported by the ELGS than EGPWS and MAP in terms of the landing spot choice and situation awareness. Although the perception and comprehension levels remained the same for all display modes, the ecological approach has helped to create an interface enhancement that increased the support of the higher levels of human cognition, i.e. projection and metacognition. This resulted in better pilot judgment regarding the landing spot choice based on the provided information about the aircraft's capabilities in relation to the terrain. The ELGS interface achieved our goal, i.e., to support pilot reasoning instead of automating and hiding it.

\section{Conclusions}

The purpose of this research was to support pilot terrain awareness by providing the functional meaning of the external constraints, imposed by the terrain, in relation to the aircraft performance limitations, the internal constraints. The experimental evaluation of the Emergency Landing Guidance System, based on an ecological approach to interface design, showed that the advantages primarily lie in supporting terrain awareness at higher levels of human cognition, thereby improving the capability of pilots to project their future status and reflect on it appropriately. 


\section{References}

Borst, C., Suijkerbuijk, H.C.H., Mulder, M. and van Paassen, M.M. (2006). Ecological Interface Design for Terrain Awareness. International Journal of Aviation Psychology, Vol. 16, No. 4, pp. 375-400.

Endsley, M.R., Bolté, B. and Jones, D.G. (2003). Designing for Situation Awareness: An Approach to User-Centered Design. SA Technologies. Taylor \& Francis Group Publishers. ISBN 0-748-40967-X.

Khatwa, R. and Roelen, A.L.C. (1999). An Analysis of Controlled Flight Into Terrain Accidents of Commercial Operators. Flight Safety Foundation Digest, Vol. 17, pp. 166-212.

McGuinness, B (1999). Situational Awareness and the Crew Awareness Rating Scale (CARS). Proceedings of the 1999 Avionics Conference, Heathrow, 17-18 November. ERA Technology Report 99-0815 (paper 4.3).

Ruijgrok, G.J.J. (1996). Elements of Airplane Performance. Delft University Press, Delft, The Netherlands. ISBN 90-6275-608-5.

Vicente, K.J. and Rasmussen, J. (1992). Ecological Interface Design: Theoretical Foundations. IEEE Transactions on Systems, Man and Cybernetics, Vol. 22, No. 4, pp. 589-606. 